B. Grinstein

P. Mende
Exact Heavy to Light Meson Form Factors in the Combined Heavy Quark, Large $N_{c}$ and Chiral Limits

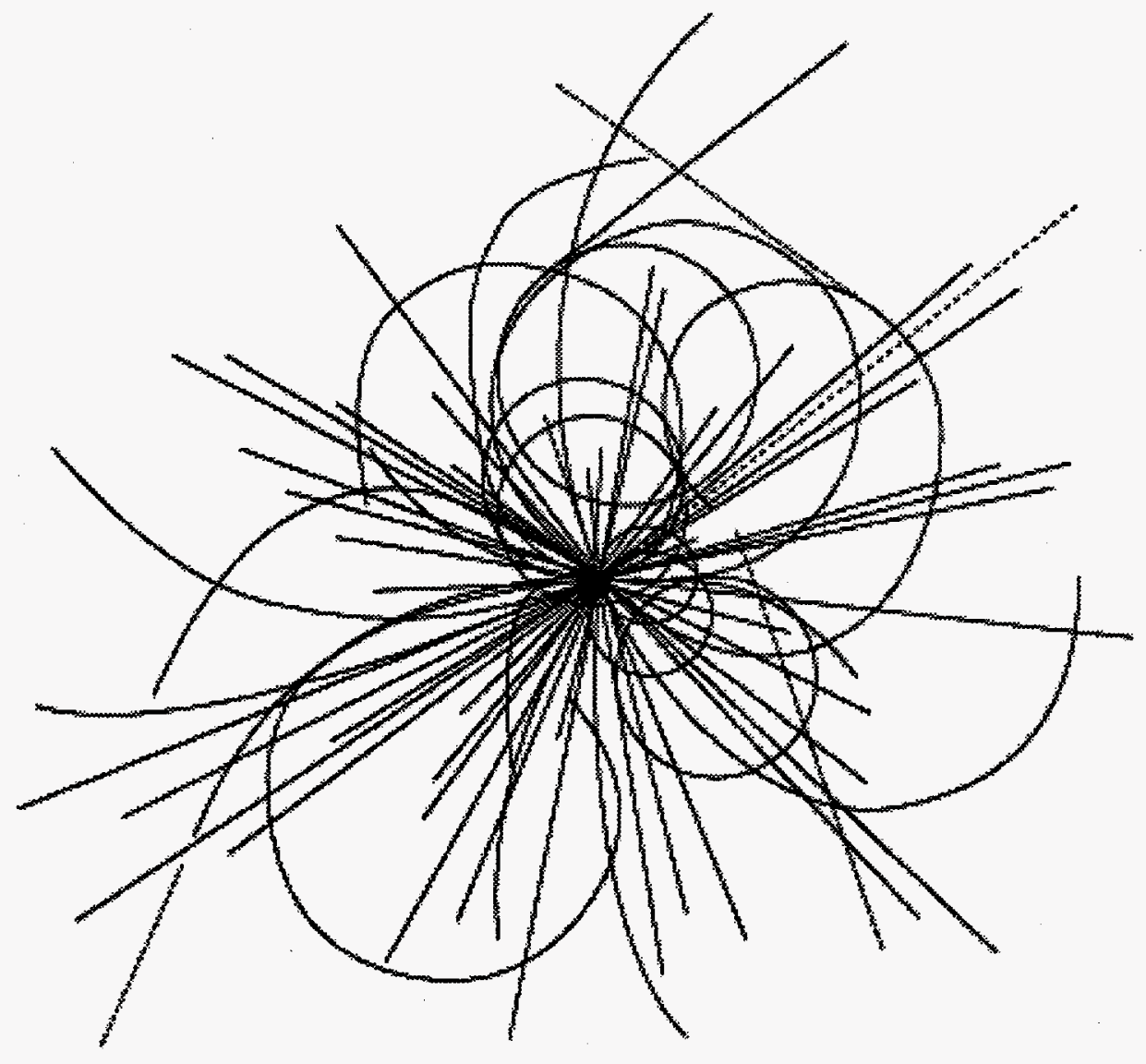

\section{Superconducting Super Collider Laboratory}

APPROVED FOR RELEASE OR

PPPRONED FOR - OR. PATENT GROUP

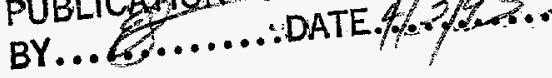




\section{Disclaimer Notice}

This report was prepared as an account of work sponsored by an agency of the United States Government. Neither the United States Government or any agency thereof, nor any of their employees, makes any warranty, express or implied, or assumes any legal liability or responsibility for the accuracy, completeness, or usefulness of any information, apparatus, product, or process disclosed, or represents that its use would not intringe privately owned rights. Relerence herein to any specific commercial product, process, or service by trade name, trademark, manufacturer, or otherwise, does not necessarily constitute or imply its endorsement, recommendation, or favoring by the United States Government or any agency thereof. The views and opinions of authors expressed herein do not necessarily state or reflect those of the United States Govemment or any agency thereot. 


\section{DISCLAIMER}

Portions of this document may be illegible in electronic image products. Images are produced from the best available original document. 


\title{
Exact Heavy to Light Meson Form Factors in the Combined Heavy Quark, Large $N_{c}$ and Chiral Limits*
}

\author{
B. Grinstein
}

Superconducting Super Collider Laboratory ${ }^{\dagger}$

2550 Beckleymeade Ave.

Dallas, TX 75237

P. Mende

Brown University

Department of Physics

Providence, Rhode Island 02912

December 1993

"Submitted to the "American Physical Society Physical Review Letters" Journal.

tOperated by the Universities Research Association, Inc., for the U.S. Department of Energy under Contract No. DE-AC35-89ER40486. 
SSCL-Preprint-544

Brown HET-928

hep-ph/9312353

\title{
Exact Heavy to Light Meson Form Factors in the Combined Heavy Quark, Large $N_{c}$ and Chiral Limits.
}

\author{
Benjamín Grinstein ${ }^{\star}$ and Paul F. Mende ${ }^{\star \star}$ \\ ^Superconducting Super Collider Laboratory, Dallas, Texas 75237

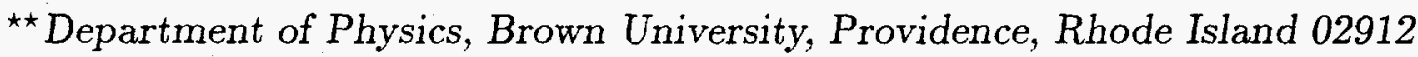

We demonstrate that the form factors of local operators between a heavy meson state (like the $B$ ) and a light pseudoscalar state (like the pion) are given exactly by a single pole form in the combined heavy quark, large $N_{\mathrm{c}}$ (number of colors) and chiral limits. We discuss the deviations from this exact result from finite heavy quark masses, non-zero light quark masses and finite $N_{c}$. We comment on some of the numerous implications of this result.

PACS numbers: $13.20 .-\mathrm{v}, 14.40 . \mathrm{Nd}, 12.15 \mathrm{Hh}, 11.15 . \mathrm{Pg}$

December 1993

* Address until February 9, 1994. Research supported in part by the Alfred P. Sloan Foundation and by the U.S. Department of Energy under contract DE-AC35-89ER40486. E-mail: ben@smuphy . physics. smu.edu

$\star \star$ Research supported in part by the TNRLC under an SSC Fellowship (\#FCFY9322) and by the U.S. Department of Energy under grant DE-AC02-76-ER03130. E-mail: mende@het. brown . edu 


\section{Introduction}

Little is known about form factors of local operators between a heavy meson like the $B$ - with quantum numbers of a single heavy quark $Q$ and a single light antiquark $\bar{q}$ - and light pseudoscalar mesons like the $\pi-K-\eta$ octet. Isgur and Wise have shown that heavy quark symmetries[1] relate several form factors[2], but nothing is known about their shape. Thus far all theoretical attempts to describe them are based on particular models of hadrons.

Surprisingly one can calculate the shape of these form factors exactly in one specific limit. We show in this letter that when one takes the leading term in a large number of colors $\left(N_{c}\right)$ expansion and simultaneously takes the leading term in the heavy quark expansion and the chiral limit, then the form factors are a single pole for all momentum transfers $t=q^{2}$,

$$
F\left(q^{2}\right)=\frac{C}{q^{2}-\mu^{2}}+\mathcal{O}\left(1 / N_{c}, \Lambda_{\mathrm{QCD}} / M_{Q}, \Lambda_{\mathrm{QCD}} / m_{q}\right) .
$$

The location of the pole is $\mu^{2}=\mu_{B^{*}}^{2}$, the squared-mass of the heavy vector meson which couples to the heavy-light current,

$$
V^{\mu}=\bar{u} \gamma^{\mu} b
$$

The constant residue $C$ is completely determined in terms of the decay constant $f_{B^{*}}$ and its coupling to a $B-\pi$ pair, $g_{B^{*} B \pi}$. To quantify the accuracy of this approximation and the expansion around this limit requires substantial exploration. This is outlined below and further details will be reported elsewhere [3].

The most immediate application of this result is to the decay

$$
\bar{B}^{0} \rightarrow \pi^{+} e^{-} \bar{\nu}_{e}
$$

which is the direct route to obtaining the elusive mixing angle $\left|V_{u b}\right|$ of the CKM matrix of the standard electroweak theory from measurements of $B$-meson decays. Little data on this mode is available yet we may anticipate the eventual measurement of the shape of its form factor as the test of these ideas.

For a thumbnail preview of the discussion below, here is the relevance of the three limits we consider: (a) in the heavy-quark limit, including $1 / M_{Q}$ corrections, the $B$ and $B^{*}$ fall into a nearly degenerate $S U(2)$ multiplet; (b) in the chiral limit, the conserved current mixes these states and suppresses transitions between multiplets; and (c) in the - large- $N_{c}$ limit multiparticle intermediate states are suppressed. 


\section{Derivation of the form factor}

Consider the matrix element of a local operator $\mathcal{O}(x)$ with quantum numbers $Q$ and $\bar{q}$ :

$$
\left\langle\pi\left(p^{\prime}\right)|\mathcal{O}(0)| \bar{B}(p)\right\rangle .
$$

For concreteness we shall speak specifically of the $\bar{B} \rightarrow \pi$ transition. In fact $\pi$ may generically stand for a light pseudoscalar meson, conveniently thought of as a $q \bar{q}$ bound

state; and $\bar{B}$ is a ground state heavy meson, that is, the lightest with $Q \bar{q}$ quantum numbers. This matrix element can be written as a sum over tensor structures times form factors, i.e., functions of the invariant momentum transfer $q^{2}=\left(p-p^{\prime}\right)^{2}$. For example, the current in (1.2) defines two form factors, $f_{+}$and $f_{-}$:

$$
\left\langle\pi\left(p^{\prime}\right)\left|V_{\mu}\right| \bar{B}(p)\right\rangle=f_{+}\left(q^{2}\right)\left(p+p^{\prime}\right)_{\mu}+f_{-}\left(q^{2}\right)\left(p-p^{\prime}\right)_{\mu} .
$$

We evaluate the left-hand side by inserting a complete set of states which couple with the same quantum numbers as the current. In the large- $N_{c}$ limit [4] the single-particle intermediate states dominate. If $F\left(q^{2}\right)$ is a form factor, then in the large- $N_{c}$ limit

$$
F\left(q^{2}\right)=\sum_{n} \frac{f_{n} g_{n B \pi}}{q^{2}-\mu_{n}^{2}}
$$

where the sum is over resonances $B_{n}$ with masses $\mu_{n}$, and couplings $f_{n}$ and $g_{n} B \pi$ to the current from the vacuum and to the $B-\pi$ pair,

$$
g_{n B \pi} \propto\left\langle\pi B_{n} \mid B\right\rangle .
$$

Note that the sum over poles eq. (2.3) is very different from the statement of eq. (1.1) that a single pole term contributes: before specifying the residues the sum may be a quite general function. This generality is amply illustrated by the case of $\bar{Q} Q$ charmonium-type states of heavy quarks where the residues of many states are large and rapidly varying[5]. The massive poles are required by the structure of excited states, yet confinement forbids the anomalous-threshold singularity at small $q^{2}$ which ought to be present to describe the large size of a non-relativistic quark distribution. Hence the electromagnetic form factor is given by exactly such a sum of poles but with rapidly varying numerator coefficients. The form factor of heavy quarkonium is therefore never well-represented by a single term of (2.3), yet is a smooth function of $q^{2}$ for all $q^{2}<\mu_{0}^{2}$. 
The lowest mass state in the sum (2.3) is either the ground state pseudoscalar meson $B$ or the ground state vector meson $B^{*}$, according to whether the local operator $\mathcal{O}$ has odd or even parity, respectively. It is obvious that this state dominates in the small kinematic region

$$
q^{2} \approx \mu_{B}^{2}
$$

to show that this state dominates over a large range of $q^{2}$ is a dynamical question that must be addressed by evaluating the behavior of the couplings $f_{n}$ and $g_{n B \pi}$. This is the question which we take up here.

As usual, the states $B$ and $B^{*}$ have equal masses in the leading order in the $1 / M_{Q}$ expansion; the leading correction is the familiar hyperfine interaction which introduces a spin-splitting, $\mu_{B^{*}}-\mu_{B}=\mathcal{O}\left(1 / M_{Q}\right)$, while $\mu_{B^{*}}^{2}-\mu_{B}^{2}=\Lambda_{0}^{2}$ independent of $M_{Q}$ in the large mass limit.

We wish to show that in the leading order in the $1 / M_{Q}$ expansion and the chiral limit $m_{q} \rightarrow 0$ the couplings $g_{n B \pi}$ vanish except for the case when $n$ corresponds to the $B^{*}$ state. Then the general expansion (2.3) reduces to the form (1.1).

Consider the how these same couplings arise in a different matrix element: let us look at the light quark axial current,

$$
a_{\nu}=\bar{q} \gamma_{\nu} \gamma^{5} q
$$

between the $B$ state and the generic resonance $B_{n}$. In the leading order in the $1 / N_{c}$ expansion it is given as a sum

$$
\left\langle B_{n}\left|a_{\nu}\right| B\right\rangle=\sum_{\ell} \frac{\left\langle 0\left|a_{\nu}\right| \pi^{(\ell)}\right\rangle\left\langle\pi^{(\ell)} B_{n} \mid B\right\rangle}{p^{\prime 2}-\mu_{\ell}^{2}} .
$$

where $\ell$ runs over single particle states $\pi^{(\ell)}$ that are produced out of the vacuum by the light quark axial current, and $\mu_{\ell}$ stands for its mass.

In the chiral limit only the pion, $\ell=0$, contributes to this sum and the result is proportional to the couplings of interest, $\left\langle\pi B_{n} \mid B\right\rangle$.

To demonstrate this let us show that, in the chiral limit,

$$
\left\langle 0\left|a_{0}\right| \pi^{(\ell)}\left(\vec{p}^{\prime}=\overrightarrow{0}\right)\right\rangle=0
$$


except for the pion. We need only consider the time component of the current in the rest frame of the state. In the chiral limit the axial current is conserved:

$$
\begin{aligned}
0 & =\left\langle 0\left|\partial_{\mu} a^{\mu}\right| \pi^{(\ell)}(\overrightarrow{0})\right\rangle \\
& =p_{\mu}^{\prime}\left\langle 0\left|a^{\mu}\right| \pi^{(\ell)}(\overrightarrow{0})\right\rangle . \\
& =\mu_{\ell}\left\langle 0\left|a_{0}\right| \pi^{(\ell)}(\overrightarrow{0})\right\rangle
\end{aligned}
$$

Thus eq. (2.7) reduces to

$$
\left\langle B_{n}\left|a_{0}\right| B\right\rangle=\frac{i f_{\pi} p_{0}^{\prime}\langle\pi B \mid n\rangle}{p^{\prime 2}},
$$

when $\vec{p}^{\prime}=0$. Using conservation of $a_{\mu}$ again gives

$$
\begin{aligned}
0 & =p_{0}^{\prime}\left\langle B_{n}\left|a^{0}\right| B\right\rangle \\
& =i f_{\pi}\left\langle\pi B_{n} \mid B\right\rangle .
\end{aligned}
$$

This means that

$$
\left\langle\pi B_{n} \mid B\right\rangle=0
$$

in the frame $\vec{p}^{\prime}=0$, but this holds generally since the matrix element is a Lorentz scalar.

To go from $\left\langle\pi B_{n} \mid B\right\rangle$ to the form factors of $B \rightarrow \pi$ decay we now must specify the spin-parity quantum numbers of the state $|n\rangle$. Consider first the case in which $B_{n}$ is a scalar. The off-shell matrix element $\left\langle\pi B_{n} \mid B\right\rangle$ can be characterized by a single 'form-factor',

$$
\left\langle\pi B_{n} \mid B\right\rangle=g^{(n)}\left(p^{\prime 2}, q^{2}, p^{2}\right) .
$$

We apply a standard dispersion relation to form factors $f_{ \pm}\left(q^{2}\right)$, which are of the form (2.3), to replace the matrix elements in the numerator by their residue at the pole, $q^{2}=\mu_{n}^{2}$. Thus to evaluate $f_{ \pm}$, only the value of the form factor on-shell is needed. But we have just shown above that

$$
g^{(n)}\left(\mu_{\pi}^{2}, \mu_{n}^{2}, \mu_{B}^{2}\right)=0 .
$$

Hence scalar $Q \bar{q}$ excited states do not contribute to the resonant sum of eq. (2.3).

Consider next what happens when $B_{n}$ is a vector. The matrix element is

$$
\left\langle\pi B_{n} \mid B\right\rangle=\left(p+p^{\prime}\right) \cdot \epsilon g_{+}^{(n)}\left(q^{2}\right)+\left(p-p^{\prime}\right) \cdot \epsilon g_{-}^{(n)}\left(q^{2}\right),
$$

where $\epsilon$ is the polarization vector of the state $B_{n}$ and its vacuum coupling through the vector current is defined by

$$
\left\langle 0\left|V_{\nu}\right| B_{n}\right\rangle=i f_{n} \epsilon_{\nu}
$$


The contribution to the $B \rightarrow \pi$ form factors is then

$$
\begin{aligned}
& f_{+}=\frac{f_{n} g_{-}^{(n)}\left(\mu_{n}^{2}\right)}{q^{2}-\mu_{n}^{2}} \\
& f_{-}=-f_{n} g_{+}^{(n)}\left(\mu_{n}^{2}\right) / \mu_{n}^{2}-\frac{f_{n} g_{-}^{(n)}\left(\mu_{n}^{2}\right) \mu_{B}^{2} / \mu_{n}^{2}}{q^{2}-\mu_{n}^{2}} .
\end{aligned}
$$

The vanishing of $\left\langle\pi B_{n} \mid B\right\rangle$, eq. (2.12), for an on-shell $B_{n}$ can now be applied to the form factors $g_{ \pm}$and therefore to $f_{ \pm}$. Nothing is learned about $g_{+}$since $B_{n}$ is on-shell. It is easy to see in the $B_{n}$ rest frame $\vec{p}=\vec{p}^{\prime}$ that

$$
g_{-}^{(n)} \vec{\epsilon} \cdot \vec{p}=0
$$

Let us restrict attention for now to the exact chiral limit in the heavy quark (infinite) mass limit. Then

$$
\vec{\epsilon} \cdot \vec{p}=|\vec{p}| \cos \theta
$$

where $\theta$ is the angle between the polarization and the momentum vectors, and is generally non-vanishing. But from the kinematics it is also true that

$$
|\vec{p}|=\frac{\mu_{n}^{2}-\mu_{B}^{2}}{2 \mu_{n}}= \begin{cases}\Lambda_{0}^{2} / 2 \mu_{B^{*}}=\mathcal{O}\left(1 / M_{Q}\right) & \text { for } n=B^{*} \\ \Lambda_{n}+\mathcal{O}\left(1 / M_{Q}\right) & \text { otherwise }\end{cases}
$$

where we introduce the mass difference $\Lambda_{n} \equiv \mu_{n}-\mu_{B}$ for $n \neq B^{*}$ states, and take the large mass limit in the last equality. Therefore, in the combined limit,

$$
0=g_{-}^{(n)}|\vec{p}|= \begin{cases}0 & \text { for } n=B^{*} \\ g_{-}^{(n)} \Lambda_{n} & \text { otherwise }\end{cases}
$$

The couplings to excited states thus go as $g_{-}^{(n)} \sim \mathcal{O}\left(1 / M_{Q}\right) \rightarrow 0$ except for $n=B^{*}$, for which $g_{-}^{\left(B^{*}\right)} \sim \mathcal{O}(1)$.

We thus obtain the advertised result that the form factor is given by the single pole:

$$
f_{+}=\frac{f_{B^{*}} g_{-}^{\left(B^{*}\right)}\left(\mu_{B^{*}}^{2}\right)}{q^{2}-\mu_{B^{*}}^{2}} \approx f_{-}
$$

The last relation follows since $f_{-}$satisfies

$$
f_{-}=-f_{+}+\left[\left(1-\mu_{B}^{2} / \mu_{B^{*}}^{2}\right) f_{+}+K\right],
$$


where $K$ is an undetermined constant. Since we have taken the large mass limit, our form factors should satisfy the standard scaling laws

$$
\begin{aligned}
& f_{+}+f_{-} \sim \mu_{B}^{-1 / 2} \\
& f_{+}-f_{-} \sim \mu_{B}^{1 / 2}
\end{aligned}
$$

and therefore the term in the square bracket in (2.22) can be neglected.

Some remarks:

(1) The choices made of particular reference frames were for convenience and not necessary to the derivation. One can obtain, for instance, the same result eq. (2.12) by taking the divergence on both sides of (2.7) and letting $p^{\prime 2} \rightarrow 0$ : every term in the sum then vanishes except for the massless state.

(2) Higher spin states can be readily incorporated into the discussion. A spin- $\ell$ meson is characterized by a totally symmetric traceless transverse $\ell$-index "polarization" tensor, $\epsilon_{\mu_{1} \ldots \mu_{\ell}}$. The generalization of eq. (2.15) has then, on the right hand, a sum over form factors

$$
\sum_{\text {sign permutations }} g_{ \pm \cdots \pm} \epsilon^{\mu_{1} \cdots \mu_{\ell}}\left(p \pm p^{\prime}\right)_{\mu_{1}} \cdots\left(p \pm p^{\prime}\right)_{\mu_{\ell}}
$$

As in the vector case, we only learn about one form factor, namely $g_{-} \ldots \ldots$. The rest of the argument is then just as before.

(3) The large- $N_{c}$ limit suppressed multiparticle intermediate states but not zero-particle intermediate states. Any contact term contribution to the form factor shows up as part of the constant $K$ and is instead suppressed by a factor of $1 / M_{Q}$.

\section{Discussion}

We computed the hadronic form factor in the triple limit of large- $N_{c}$, heavy quarks, and chiral symmetry. We predict the weak decay form factor governing $\bar{B}^{0} \rightarrow \pi^{+} e^{-} \bar{\nu}_{e}$ to be pole-dominated as in the result eq. (2.21) for all $q^{2}$. Moreover, the two factors in the numerator, $f_{B^{*}}$ and $g^{\left(B^{*}\right)}$, can be roughly estimated at least by scaling measured values for charmed mesons using heavy quark symmetry.

It is crucial to trace how the three limits $\left(1 / N_{c} \rightarrow 0,1 / M_{Q} \rightarrow 0\right.$ and $\left.m_{q} \rightarrow 0\right)$ entered our derivation, for the next goal must be to estimate the corrections and understand the approach to the limit. Is the result independent of the order of the limits? If not, what limit and approximations are appropriate? 
There is a subtle issue in the ordering of the approach to the simultaneous $1 / M_{Q} \rightarrow 0$ and $m_{q} \rightarrow 0$ limits. In ref. [6], for instance, Isgur and Wise examined the effect of the $B^{*}$ pole on the $B \rightarrow \pi$ transition. They concluded that pole-dominance held only for a narrow kinematic region in contrast with our result. They relied crucially on taking the pion mass to zero first rather than, say, holding $\mu_{\pi} \mu_{B}$ fixed. Consider small chiral symmetry breaking corrections to eq. (2.12): for infinitesimal $m_{q}$,

$$
\left\langle\pi B \mid B^{*}\right\rangle \sim m_{q} \varphi\left(M_{Q}, m_{q}, \Lambda\right)
$$

where $\varphi$ is some function of the masses and the hadronic scale $\Lambda$, and $m_{q} \varphi \rightarrow 0$ as $m_{q} \rightarrow 0$. For an on-shell $B^{*}$ this implies

$$
g_{-}^{\left(B^{*}\right)} \Lambda_{0}^{2} / M_{Q} \sim m_{q} \varphi\left(M_{Q}, m_{q}, \Lambda\right)
$$

Therefore, the function $\varphi$ has a finite limit as $m_{q} \rightarrow 0$ for $M_{Q}$ fixed ( $\Lambda$ is always fixed), while $\lim _{M_{Q} \rightarrow \infty} \varphi \sim 1 / m_{q}$.

Examples of such functions are easy to come by. A class of such functions is, for example,

$$
\varphi\left(M_{Q}, m_{q}, \Lambda\right) \propto \frac{M_{Q}^{n-1}}{\Lambda^{n+1}+m_{q} M_{Q}^{n}}
$$

for any $n>1$. The expansion parameter around the point $1 / M_{Q}=0$ is $\Lambda^{n+1} / m_{q} M_{Q}^{n}$. This type of expansion is familiar from calculations in chiral perturbation theory for heavy mesons, where one often finds[7,8] corrections to be functions of $\Lambda_{0}^{2} / \mu_{\pi} \mu_{B} \sim$ $\Lambda_{\mathrm{QCD}}^{3 / 2} / m_{q}^{1 / 2} M_{Q}$. Just as the example eq. (3.3), the corrections computed in refs. $[7,8]$ are not singular in either of the limits $m_{q} \rightarrow 0$ or $1 / M_{Q} \rightarrow 0$, but the expansion parameters for the expansions about $m_{q}=0$ and $1 / M_{Q}=0$ are the inverse of each other. We see that the large mass and chiral limits are inextricably coupled.

Let us consider one of these calculations in some detail. Chiral perturbation theory can be used to predict the leading corrections to the form factors for semileptonic $B \rightarrow D$ or $D^{*}$ decays which are generated at low momentum, below the chiral symmetry breaking scale. Deviations from the predicted normalization of form factors arise from terms of order $1 / M_{Q}^{2}$ in either the lagrangian or the current as dictated by non-perturbative physics, and there are computable corrections that arise from the terms of order $1 / M_{Q}$ in the lagrangian 
which enter at one-loop. Retaining only the dependence on the larger hyperfine splittings $\Delta_{D}=\mu_{D^{*}}-\mu_{D}$, the correction to the matrix elements at zero recoil are[7]

$$
\left\langle D(v)\left|J_{\mu}^{\bar{c} b}\right| B(v)\right\rangle=2 v_{\mu}\left(1-\frac{3 g^{2}}{2}\left(\frac{\Delta_{D}}{4 \pi f}\right)^{2}\left[F\left(\Delta_{D} / \mu_{\pi}\right)+\ln \left(\mu^{2} / \mu_{\pi}^{2}\right)\right]+C(\mu) / m_{c}^{2}\right)
$$

where $C$ and stands for tree level counter-terms and

$$
F(x) \equiv 2 \int_{0}^{\infty} d z \frac{z^{4}}{\left(z^{2}+1\right)^{3 / 2}}\left(\frac{1}{\left[\left(z^{2}+1\right)^{1 / 2}+x\right]^{2}}-\frac{1}{z^{2}+1}\right)
$$

This matrix element has alternate, inequivalent expansions around the limits $x=0$ and $x=\infty$, which correspond to individually taking $M_{Q} \rightarrow \infty$ and $\mu_{\pi} \rightarrow 0$, respectively. For small $x, F(x) \sim x$ while as $x \rightarrow \infty, F(x) \sim \log x$ and this cancels the $\log$ singularity above. It is instructive to compare the behavior of $g_{-}^{\left(B^{*}\right)}$ to that of the form factors of higher vector states, $g_{-}^{(n)}$. For these we expect a relation similar to $(3.2)$,

$$
g_{-}^{(n)} \Lambda_{n} \sim m_{q} \varphi\left(M_{Q}, m_{q}, \Lambda\right)
$$

where $\varphi$ is not necessarily the same function as above, but has the same properties. We therefore predict that at finite small $m_{q}$ one should find

$$
g_{-}^{(n)} / g_{-}^{\left(B^{*}\right)} \sim 1 / M_{Q}
$$

Consider the strong decays of the excited states $D_{1}^{\prime}$ and $D_{2}^{*}$ into the $D$ or $D^{*}$ mesons and a pion studied in ref. [9], as well as the corresponding states where charm is replaced by bottom. In the notation of ref. [9] these strong decays are described in the combined heavy quark and chiral limits by the effective (chiral) lagrangian

$$
\mathcal{L}_{d}=\frac{h_{1}}{\Lambda_{\chi}} \operatorname{Tr}\left[\bar{H}_{a} T_{b}^{\mu}\left(i D_{\mu}\right)_{b a} \gamma_{5}\right]+\frac{h_{2}}{\Lambda_{\chi}} \operatorname{Tr}\left[\bar{H}_{a} T_{b}^{\mu}\left(i \not D A_{\mu}\right)_{b a} \gamma_{5}\right]+\text { h.c. }
$$

where $T^{\mu}$ and $H$ are the spin supermultiplets containing the $D_{1}^{\prime}$ and $D_{2}^{*}$, and $D$ and $D^{*}$ fields, respectively, and $A_{\mu}$ is the axial vector field. It follows that in the large- $N_{c}$ limit one must have $h_{1}+h_{2} \sim \mathcal{O}\left(1 / M_{Q}\right)$. This is nontrivial information which is not automatically included in the effective lagrangian formulation.

In contrast to the expectation that $g_{B^{*} B \pi}$ scales like $M_{Q}$, it has been shown above that $g_{B * B \pi} \sim 1$. Note that this is necessary if the form factors in eq. $(2.21)$ are to scale 
according to (2.23) over the whole kinematic range. Recall that $f_{B^{*}} \sim M_{Q}^{1 / 2}$, and consider the region of maximum momentum transfer $q^{2} \approx q_{\max }^{2} \equiv\left(\mu_{B}-\mu_{\pi}\right)^{2}$. In the chiral limit,

$$
f_{+}\left(q_{\max }^{2}\right)=\frac{f_{B^{*}} g_{B^{*} B \pi}}{\Lambda_{0}^{2}}+\cdots
$$

where the dots indicate contributions of states above the $B^{*}$. As mentioned above, Isgur and Wise have argued that $f_{ \pm}$should be pole-dominated in the proximity of $q_{\max }^{2}$ in the chiral limit[6]. They observe that, were $g_{B^{*} B \pi}$ to scale like $M_{Q}$, the $B^{*}$-pole contribution would scale like $M_{Q}^{3 / 2}$, while other resonant contributions (and the continuum) would scale like $M_{Q}^{1 / 2}$ even if their couplings to the $B-\pi$ pair were to scale also as $M_{Q}$. At least in the large- $N_{c}$ limit, this is not the case at all. $f_{ \pm}$never violate the scaling laws (2.23), and pole dominance occurs because the couplings to higher resonances are suppressed by a power of $M_{Q}$.

The large- $N_{c}$ expansion was used twice to write matrix elements as discrete sums over single resonances, in eqs. (2.3) and (2.7). The essential point was not so much the precise form of the sum but rather the absence, or suppression, of smooth background contributions. We do know that the large- $N_{c}$ limit appears at least as good for heavy meson as for light mesons [11].

It is tempting to conjecture that $g_{B^{*} B \pi} \sim 1$ even at finite $N_{c}$. This would avoid the contradiction with the scaling laws for $f_{ \pm}$. This scaling behavior of $g_{B^{*} B \pi}$ is important to applications where the naive scaling is used to compare $B$ and $D$ meson couplings. In the language of the effective chiral lagrangian of ref. [10], the coupling $g$ of the $B$ - $B^{*}$ multiplet to the pion axial current scales like $1 / M_{Q}$ rather than $M_{Q}^{0}$.

Both as a check on the assumptions discussed here and as a concrete laboratory for exploring the expansion around the limit we have analyzed the predictions of this work in the 't Hooft model, QCD in $1+1$ dimensions to leading order in the large $-N_{c}$ expansion. Details of this work will be extensively discussed elsewhere[3]. In this exactly solvable model, we have shown that the single pole picture is stable as one tunes the heavy quark mass down from infinity and independently stable as one tunes the light quark mass up from zero. In two dimensions there is no spin, of course, so there are no vector mesons. Instead the $B$-meson couples directly to the vector current and therefore plays the role of the $B^{*}$-meson of the preceding discussion. We show precisely that the couplings $g_{n} B \pi$ vanish as $m_{q} \rightarrow 0$ for $n \neq B$ but not for $n=B$. Moreover, this single pole dominance holds for any heavy quark mass $M_{Q}$. 
It is apparent that the result can be generalized in several directions. For example, one may consider the form factor for a matrix element of a local operator between a pion and non-ground state $Q \bar{q}$ meson. Take, for example, the $B_{1}$ and $B_{2}^{*}$ mesons, which form a multiplet of heavy quark spin symmetry. The above result states that, modulo accidentally degenerate states, the form factors are pole dominated by a pole at the $B_{1}$ or $B_{2}^{*}$ squared-mass. One may consider other states for which the conserved axial current is a good interpolating field, e.g., the $A_{1}$ pseudo-vector meson. Perhaps more interestingly, one may instead consider other conserved currents as interpolating fields. The first obvious candidate is the vector current $\bar{q} \gamma^{\mu} q$. This can be used as interpolating field for the vector mesons, like the $\rho$ and $K^{*}$.

Acknowledgments: We are indebted to Sidney Coleman for an incisive question and comment which led to this work and we thank William Bardeen for helpful discussions. 


\section{References}

[1] N. Isgur and M.B. Wise, Phys. Lett. B232 (1989) 113;

N. Isgur and M.B. Wise, Phys. Lett. B237 (1990) 527

[2] N. Isgur and M.B. Wise, Phys. Rev. D42 (1990) 2388

[3] B. Grinstein and P.F. Mende, in preparation

[4] G. 't Hooft, Nucl. Phys. B72 (1974) 461

[5] R.L. Jaffe and P.F. Mende, Nucl. Phys. B369 (1992) 189

[6] N. Isgur and M.B. Wise, Phys. Rev, D41 (1990) 151

[7] L. Randall and M.B. Wise, Phys. Lett. B303 (1993) 135, hep-ph/9212315

[8] A. Falk and B. Grinstein, $\bar{B} \rightarrow \bar{K} e^{+} e^{-}$in Chiral Perturbation Theory, hepph/9306310, SLAC-PUB-6237, SSCL-Preprint-484, June 1993;

B. Grinstein, Phys. Rev. Lett. 71 (1993) 3067

[9] A. F. Falk and M. Luke, Phys. Lett. B292 (1992) 119, hep-ph/9206241

[10] G. Burdman, J. F. Donoghue, Phys. Lett. B280 (1992) 287;

M. B. Wise, Phys. Rev. D45 (1992) 2188;

T.-M. Yan, H.-Y. Cheng, C.-Y. Cheung, G.-L. Lin, Y. C. Lin and H.-L. Yu, Phys. Rev. D46 (1992) 1148

[11] B. Grinstein and P.F. Mende, Phys. Rev. Lett. 69 (1992) 1018, hep-ph/9204206; M. Burkardt and E.S. Swanson, Phys. Rev. D46 (1992) 5083 